\title{
Cornual pregnancy-an unusual site of pregnancy: a case report and literature review
}

\author{
Chowdhury TS ${ }^{\mathrm{a}}$, Hasan $\mathrm{H}^{\mathrm{b}}$, Chowdhury TA
}

\begin{abstract}
Ectopic pregnancy is a condition where gestational sac is located outside the uterine cavity. Cornual pregnancy, also known as interstitial pregnancy, is a rare type of ectopic pregnancy that develops in the interstitial portion of the fallopian tube and invades through the uterine wall. It poses great diagnostic challenge because of its unusual presentation and late diagnosis. Cornual pregnancy, if not diagnosed early, may present with massive and uncontrollable bleeding even leading to maternal death. We hereby report an unusual presentation of cornual pregnancy which was diagnosed and subsequently managed successfully.
\end{abstract}

Key words: cornual pregnancy, ectopic pregnancy, fallopian tube.

(BIRDEM Med J 2020; 10(1): 64-67)

\section{Introduction}

Implantation of fertilized ovum in sites other than the normal uterine cavity is called ectopic pregnancy; commonest site being the fallopian tube. Fallopian tube has different parts which vary in diameter and muscular strength. The commonest site of ectopic pregnancy is in the ampullary region of the fallopian tube as this is the most distensible part where fertilization of ovum and sperm takes place. The interstitial part of the fallopian tube, measuring $1.2 \mathrm{~cm}$ in length and $0.7 \mathrm{~cm}$ in width is situated within the uterine wall musculature. Pregnancies implanted in this site are called interstitial (cornual) pregnancies. ${ }^{1}$ Cornual pregnancy, a rare variety of ectopic pregnancy, seen in about $2-4 \%$ of ectopic pregnancies. ${ }^{2}$ Because of myometrial distensability, they tend to present relatively late, at 7-

Author information

a. Tanzeem S Chowdhury, Associate Professor, Department of Obstetrics and Gynaecology, BIRDEM General Hospital and Ibrahim Medical College, Dhaka, Bangladesh.

b. Homaira Hasan, Resident Medical Officer, Department of Obstetrics and Gynaecology, BIRDEM General Hospital, Dhaka, Bangladesh.

c. TA Chowdhury, Chief Consultant and Professor, Department of Obstetrics and Gynaecology, BIRDEM General Hospital and Ibrahim Medical College, Dhaka, Bangladesh.

Address of correspondence: Tanzeem S Chowdhury, Associate Professor, Department of Obstetrics and Gynaecology, BIRDEM General Hospital and Ibrahim Medical College, 122, Kazi Nazrul Islam Avenue, Shahbag, Dhaka-1000, Bangladesh. Email: tanzeemsc@gmail.com

Received: August 22, 2019
12 weeks of gestation. Rupture of cornual pregnancy may result in severe hemorrhage and shock, with high mortality rate, ranging between $2-2.5 \%{ }^{2}$ According to the Confidential Enquiry into Maternal and Child Health (CEMACH) report published by Royal College of Obstetricians and Gynecologists(RCOG) press, London, United Kingdom in 2002, there were 11 deaths from ruptured ectopic pregnancy among which 7 were located in the extra uterine tube and 4 in the interstitial portion of the tube (cornual pregnancy). ${ }^{3}$ Even though, cornual pregnancies are rare but they pose a significant diagnostic and therapeutic challenge and carry a greater maternal mortality risk than pregnancy in other parts of the fallopian tube. Here we present a case report of a patient with cornual pregnancy who was admitted in BIRDEM 2 Hospital with13+ weeks of amenorrhea where previous investigation reports of early weeks missed the diagnosis.

\section{Case report}

A 27-year-old, P-1+0, non-diabetic, normotensive house wife presented with severe per vaginal bleeding for 6 hours following $13+$ weeks of amenorrhea. She had a previous uneventful pregnancy 4 years back and her child was born by Cesarean section without any complications.

History revealed that at her 6 weeks of amenorrhea she had a transvaginal sonography (TVS) which reported a case of missed abortion. For that she underwent dilatation and curettage in a local hospital but there was 
still persistence of her per vaginal bleeding following the procedure. She was advised for estimation of serum $\beta$-human chorionic gonadotropin (hCG) which was 88.6mIU/ml(ref: 1,080- 56,500mIU/ml at 6 weeks).

After one week, ultrasonography was repeated when she was diagnosed as a case of ectopic pregnancy and had a single dose of injection methotrexate (50mg) intramuscularly (IM) as medical management of ectopic pregnancy. She was advised for laparotomy as her symptoms persisted but she refused and went to abroad for further management.There she got another three doses of IM injection of methotrexate but still her per vaginal bleeding continued. Then she got admitted into BIRDEM 2 Hospital for further management.

On admission, she was severely anemic.Vital signs were within normal physiological limit. Per abdominal examination revealed no significant findings. On local vaginal examination, there was moderate per vaginal bleeding and blood was coming from the uterine cavity.

On investigations, her haemoglobin was $7.7 \mathrm{gm} / \mathrm{dl}$ on admission. Other hematological investigations were well within normal limit. Routine examination of urine showed plenty of red cells. Serum $\beta$-hCG was $14.8 \mathrm{mIU} /$ $\mathrm{ml}$. Ultrasonography of lower abdomen and pelvis showed bulky uterus with right sided cornual pregnancy (Figure 1).

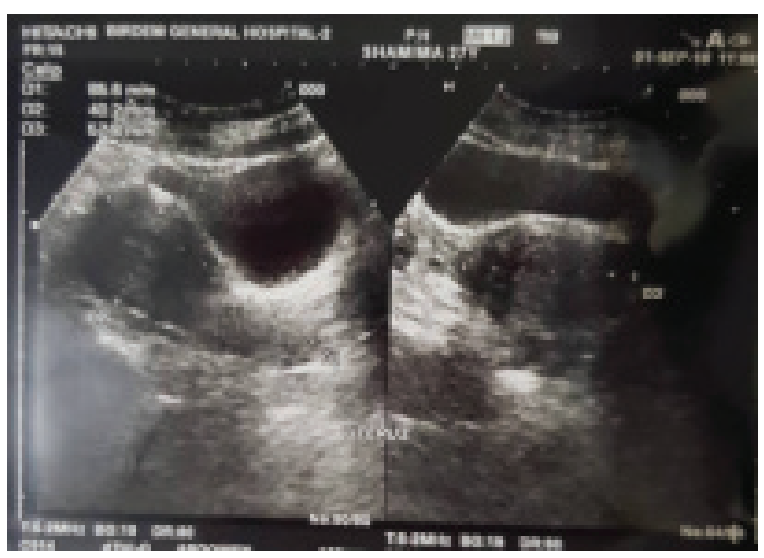

Figure 1 Ultrasonography showing cornual pregnancy

After counseling of patient and her attendant, decision for hysteroscopy followed by laparoscopy was taken. Patient was also counseled for laparotomy if necessary.

In hysteroscopy, good view of endometrial cavity was obtained. Both tubal ostia were seen. Right ostium was narrow but left ostium was normal.
Then laparoscopy was done. Pelvic cavity was free from adhesions.Uterus was asymmetrically enlarged with a highly vascular bulging on the right cornu which appeared to be a case of cornual pregnancy.

Due to high vascularity, decision for laparotomy was taken. Both tubes and ovaries looked apparently healthy. After preliminary infiltration with diluted vasopressin solution. Incision was made over the bulging and degenerated gestational sac, placental tissue and organized blood clots were removed (Figure 2).Then uterus was closed in layers.

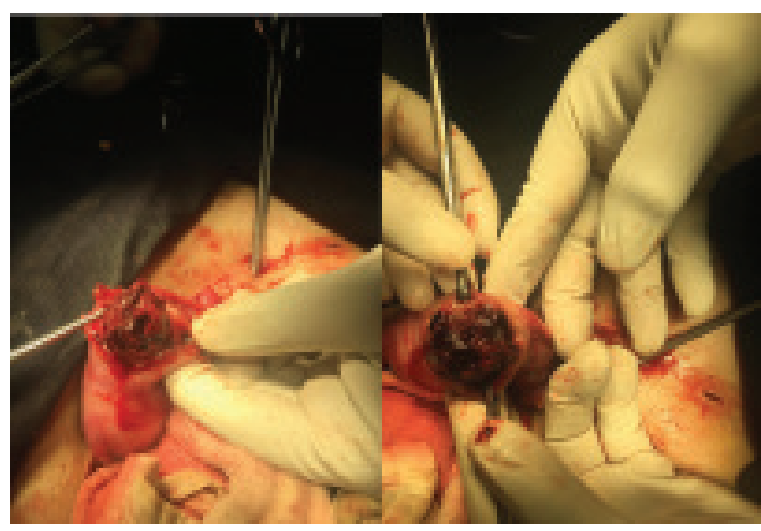

Figure 2 Per operative pictures of cornual pregnancy

Microscopic examination of the removed tissue showed, necrosed tissues, blood clots and degenerated as well as well-preserved chorionic villi lined by trophoblastic cells. Post-operatively the patient was stable and discharged on the fourth post-operative day without any complication.

\section{Discussion}

Cornual ectopic pregnancy is a rare type of tubal ectopic with a high risk of rupture and hemorrhage compared to other types of ectopic pregnancy. ${ }^{4}$ It occurs within the interstitial portion of the fallopian tube and therefore has the potential to grow to larger sizes compared to other types of tubal pregnancies by the time they present. The risk of cornual pregnancy is increased in patients with history of pelvic inflammatory disease or tubal surgery or conception after tubal ligation. Rare associations may be seen when there is history of salpingectomy or salpingostomy. It can also occur after assisted reproductive technique specially if there is difficulty during the embryo transfer procedure. ${ }^{5}$ 
Clinical presentation depends on whether pregnancy sac has ruptured or not. Unruptured cases may present with history of repeated abdominal pain at few days interval and per vaginal bleeding. Ruptured cases usually present with severe abdominal pain and features of shock. Cornual pregnancies are associated with the highest risk of massive, uncontrollable bleeding which can happen at any time in early pregnancy, thus leading to relatively high mortality rate. ${ }^{6}$ Some authors have reported rupture of the interstitial pregnancy followed by formation of a hematoma in the broad ligament. ${ }^{7}$

Ultrasonography is the most commonly used tool where it has $80 \%$ sensitivity and $98 \%$ specificity. The diagnostic criteria includes: a) Absence of gestational sac in uterine cavity, b) Gestational sac seen independently and less than $1 \mathrm{~cm}$ from the lateral edge of the uterine cavity, c) Thin layer of myometrium around the gestational sac, d) Interstitial line sign (echogenic line extending to the gestational sac): During early days of cornual gestation, the sac is located in the lateral part of the uterus. Later on, the gestational sac shift above the uterine fundus. Thus, cornual pregnancy detected late may appear as an eccentric uterine pregnancy ${ }^{8,9}$ Transvaginalsonography (TVS) is much better in diagnosis of cornual pregnancy than transabdominal sonography and one study has shown that early diagnosis of cornual pregnancy with TVS allows for first trimester conservative management with methotrexate. ${ }^{10}$ Magnetic resonance imaging (MRI) may also be an important tool to reveal the eccentric location of gestational sac to the junctional zone but it is more expensive. ${ }^{11}$

Early recognition of the cornual pregnancy is essential for medical management of cornual pregnancy. It can be used if there is hemodynamic stability, no medical contraindications to methotrexate and no signs of rupture. The modes for administration of methotrexate (dose: $1 \mathrm{mg} / \mathrm{kg}$ ) include systemic, laparoscopic, transvaginal, and with ultrasound guidance. According to RCOG guidelines, medical treatment should be given only when levels of $\beta \mathrm{hCG}$ are less than $3000 \mathrm{IU} / \mathrm{L}$ and symptoms are minimal. The systemic route of administration is a safe and highly effective treatment and offers advantages over local injection in that it is less invasive and not operator dependent.On the other hand, advantage of local administration of methotrexate has been its favorable side-effect profile and low
dosage.Medical management with methotrexate may not always be successful and if treatment fails, surgical intervention may be required. Other non-surgical methods include selective uterine artery embolisation associated with or after methotrexate failure which can be used successfully in treating selected cases of early cornual pregnancy. ${ }^{12}$

Traditionally, the treatment of cornual pregnancy has been surgical management which includes corneal resection, cornuostomy and hysterectomy. Laparotomy is necessary in patients with ruptured cornual pregnancy with profuse and life threatening haemorrhage. ${ }^{13}$ In other cases, laparoscopic cornual resection, laparoscopic cornuostomy or hysteroscopic removal of interstitial ectopic tissue can be done. ${ }^{14}$ But conservative surgical approach may lead to catastrophic hemorrhage, so ipsilateral uterine artery ligation can be performed before attempting to repair a ruptured uterine cornu which will help to achieve homeostasis and allow time to repair the cornu. ${ }^{15}$

The size of cornual gestation determines the feasibility of laparoscopic approach. Laparoscopicsalpingostomy may be appropriate for gestation less than $3.5 \mathrm{~cm}^{1}$ and cornual resection may be preferred for gestation of more than $4 \mathrm{~cm} .{ }^{16} \mathrm{~A}$ laparoscopic approach should only be attempted if the surgeon is skilled in laparoscopic techniques and has the ability to convert the operation quickly to a laparotomy. ${ }^{16}$

Hysteroscopic treatment is used in cases of noncompliance with treatment with methotrexate or who did not respond to management with methotrexate. With this technique, cornual endometrium is removed (including tubal ostium) and pregnancy sac extracted under laparoscopic guidance. ${ }^{17}$

In contrast with other types of ectopic pregnancy, expectant management of corneal pregnancy is considered unsafe because of high risk of complications, which include uterine rupture and massive internal bleeding.

Following successful management of cornual pregnancy, there are some concerns regarding future pregnancy. Surgical treatment involving resection of the involved cornual region is associated with decreased fertility rates and increased rates of uterine rupture in future pregnancies. ${ }^{14} \mathrm{So}$, it is mostly agreed that caesarean 
section should be the optimum mode of delivery for all pregnancies following cornual pregnancy. ${ }^{13}$ The second concern after conservative management of cornual pregnancy is recurrence of ectopic pregnancy, particularly cornual pregnancy on the same side. So, appropriate counseling regarding future pregnancy risks and optimum mode of delivery should be done before discharge from the hospital.

\section{Conclusion}

Cornual pregnancy poses a significant diagnostic and therapeutic challenge and carries a greater maternal mortality risk than other types of tubal pregnancy.So, the purpose of this paper is to increase awareness and understanding the seriousness of cornual pregnancies and to advocate for early clinical diagnosis aided by ultrasound or lapaprosocpy.

Conflict of interest: Nothing to declare.

\section{References}

1. Tulandi T, Al-Jaroudi D. Interstitial pregnancy: results generated from the Society of Reproductive Surgeons Registry. Obstet Gynecol 2004; 103:47-50.

2. Mamatha S, Chaitra S, Ahsa S, Neha S, Nisha S. A rare case report of Cornual Pregnancy. Obstet Gynecol Int J 2016;5(6): 00179 .

3. Confidential Enquiry into Maternal and Child Health. Why Mothers Die 2000-2002. The Sixth Report of the Confidential Enquiries into Maternal Deaths in the United Kingdom. London: RCOG Press; 2004.

4. Moawad NS, Mahajan ST, Moniz MH, Taylor SE, Hurd WW. Current diagnosis and treatment of interstitial pregnancy. Am J Obstet Gynaecol 2010; 202(1): 15-19.

5. Agarwal SK, Wisot AL, Garzo G, Meldrum DR. Cornualpregnancies in patients with prior salpingectomy undergoing in vitro fertilization and embryo transfer. Fertil
Steril 1996; 65:659-60.

6. Alam IP. Cornual Pregnancy: A Case Report. Faridpur Med Coll J 2013; 8(2): 102-104.

7. Abbas AM, Sheha AM, Ali SS, Maghraby AM, Talaat E. A rare presentation of ruptured interstitial ectopic pregnancy with broad ligament hematoma: A case report. Middle East Fertility Society Journal 2017;22(1):80-83.

8. Alagbe OA, Adeniyi TO, Abayomi OA, Onifade EO. Interstitial ectopic pregnancy: a case report. Pan African Medical Journal 2017; 28:135.

9. Rizk B, Abuzeid M, Rizk C, Owens S,LaFleur J. Ectopic Pregnancy. In:Rizk B (Ed.), Ultrasound in reproductive medicine and infertility. Cambridge University Press, Cambridge, 2010. pp. 259-270.

10. Auslender R, Arodi J, Pascal B, Abramovici H. InterstitialPregnancy: Early Diagnosis by Ultrasonography. Am J Obstet Gynecol 1983;146(6): 717-718.

11. Jermy K, Thomas J, Doo A, Bourne T. The conservative management of interstitial pregnancy. BJOG 2004; 111:1283-8.

12. Deruelle P, Lucot JP, Lions C, RobertY. Management of interstitial pregnancy using selective uterine artery embolization. ObstetGynecol 2006; 107:427-8.

13. Grimbizis GF, Tsalikis T, Mikos T, Zepiridis L, Athanasiadis A, Tarlatzis BC, et al. Case report: laparoscopic treatment of a ruptured interstitial pregnancy. Reprod Biomed Online 2004; 9:447-51.

14. Ross R, Lindheim SR, Olive DL, Pritts EA. Cornual gestation: a systematic literature review and two case reports of a novel treatment regimen. J Minim Invasive Gynecol 2006; 13:748.

15. Khawaja N, Walsh T, Gill B. Uterine artery ligation for the management of ruptured cornual ectopic pregnancy. EurJObstetGynecolReprodBiol 2005; 118:269.

16. Attia M, Karuppaswamy J, Griffith H. Management of interstitial (cornual) pregnancy at 17 weeks' gestation: conservation of a ruptured uterus. J Obstet Gynaecol 2005; 25:722-3.

17. Faraj R, Steel M.Management of Cornual (Interstitial) Pregnancy. Obstet Gynaecol 2007;9: 249-55. 